\title{
A novel CNS embryonal tumor successfully treated with multi-modal therapy highlights limitation of methylation-based tumor classification
}

Ritu Lamichhane ${ }^{1}$, Paromita Roy ${ }^{1}$, Cynthia Hawkins ${ }^{2}$, Lateef Zameer ${ }^{1}$, Anisha Gehani ${ }^{1}$, Rimpa Achari ${ }^{1}$, Ben $\mathrm{Ho}^{3}$, Annie Huang ${ }^{3}$, Uri Tabori ${ }^{4}$, Vijay Ramaswamy ${ }^{5}$, Reghu Sukumaran $^{1}$, and Anirban Das ${ }^{1}$

${ }^{1}$ Tata Medical Center

${ }^{2}$ The Hospital for Sick Children

${ }^{3}$ Hospital for Sick Children Research Institute

${ }^{4}$ The Hospital for Sick Children , toronto

${ }^{5}$ Hospital for Sick Children

November 1, 2021

\section{LETTER TO THE EDITOR}

A novel CNS embryonal tumor successfully treated with multi-modal therapy highlights limitation of methylation-based tumor classification

Ritu Lamichhane ${ }^{1}$, Paromita Roy ${ }^{1}$, Cynthia Hawkins ${ }^{2}$, Lateef Zameer ${ }^{1}$, Anisha Gehani ${ }^{1}$, Rimpa B.Achari ${ }^{1}$, Ben $\mathrm{Ho}^{2}$, Annie Huang ${ }^{2}$, Uri Tabori ${ }^{2}$, Vijay Ramaswamy ${ }^{2},{ }^{*}$ Reghu K. Sukumaran ${ }^{1},{ }^{*}$ Anirban Das ${ }^{1,2}$

1 Tata Medical Center, Kolkata, India; ${ }^{2}$ Hospital for Sick Children (SickKids), Toronto, Canada

* RKS and AD jointly supervised this work

No. of pages: 7

Tables: 0, Figures: 1

Short title: Novel CNS embryonal tumor

Word count: 514

Keywords: CNS-neuroblastoma, PNET, methylation classifier, MGMT

Competing interest: nil

\section{Address for correspondence}

Dr. Anirban Das MBBS, MD, DM

Consultant, Department of Pediatric Hematology-Oncology, Tata Medical Center

Clinician Scientist Training Program, Hospital for Sick Children (SickKids), Toronto

555 University Avenue, Toronto, Ontario, Canada M5G 1X8

Email: anirban.das13@gmail.com, anirban.das@sickkids.ca 
Phone: +1-437-984-8489

\section{ABBREVIATIONS IN TEXT}

\begin{tabular}{ll}
\hline CNS & Central nervous system \\
\hline WHO & World health organization \\
CSF & Cerebrospinal fluid \\
PET & Positron emission tomography \\
MGMT & O(6)-Methylguanine-DNA-methyltransferase \\
ETMR & Embryonal tumor with multilayered rosettes \\
CNA & Copy number aberration \\
MC & Micro-RNA cluster \\
\hline
\end{tabular}

To the editor,

Methylation-based classification stratifies supratentorial embryonal tumors into 4 distinct entities, among which, CNS-neuroblastoma, FOXR 2-activated, and neuroepithelial tumors with BCOR -alteration, have been included in the new WHO classification ${ }^{1,2}$. While the tool has emerged as an important adjunct to tumor characterization in the modern era, its performance and scope may be limited by the data used to train the classifier. We share our challenges in characterizing a supratentorial embryonal tumor that was ultimately successfully treated according to its histology.

A 5-year-old boy presented with right-focal weakness for 8 -months. A $8 \times 7 \times 6.5$-cc mass in the leftfrontoparietal region (Fig.1A) was completely resected and diagnosed as CNS-neuroblastoma (Fig.1B,C). Spine, CSF and whole-body PET were clear. Methylation-based classifier failed to characterize this tumor. Unsupervised clustering grouped it closest to embryonal tumors with multi-layered rosettes (ETMR) (Fig.1D). No multi-layered rosettes were detected, LIN28 was negative, INI-1 and mismatch-repair stains were retained. Copy number aberrations (CNA) included losses in chromosomes 3p, 9 (including the $C D K N 2 A / B$ locus), 11q (partial) and 19q (partial) and focal gains on chromosome-19 (Fig.1E), but no amplification of the chromosome-19 microRNA-cluster (19MC) (Fig.1F). MYC or MYCN were not amplified. MGMT promoter was methylated (Fig.1G). Repeated attempts at sequencing failed. He was treated with craniospinal irradiation (23.4Gy, 13-fractions; tumor-bed boost 14.4Gy, 8-fractions). Adjuvant chemotherapy included vincristine, cisplatin and cyclophosphamide. The child remains in complete remission after 2-years.

CNS-neuroblastoma presents at a median age of 5-8 years, shows female preponderance, and predilection for the fronto-parietal region ${ }^{3,4}$. Radiology is heterogeneous, frequently including solid-cystic components with central necrosis and haemorrhage. Methylome analysis is the method of choice for diagnosis ${ }^{5}$. CNAs include 1q gain, aberrations/losses in 16q, 3p and 6q, and gains in chromosomes 17q and $8^{1,5,4}$. Recent analyses incorporating accurate molecular analyses suggest 5 -year survival $>80 \%{ }^{5}$. In contrast, ETMR is a tumor of infants and younger children, characterized by multi-layered pseudo-rosettes, expression of LIN28, amplification of $\mathrm{C} 19 \mathrm{MC}$ and dismal outcomes ${ }^{6,7}$. CNAs include gain in chromosome 2 and 1q, and losses in 6q. Rare $(<10 \%)$ subsets lacking C19MC amplification cluster with other ETMRs on methylome-analysis and are enriched for DICER1 variants (chromosome-14), or harbour amplifications in other micro-RNA clusters on chromosome- $13^{6}$.

The embryonal tumor in our patient failed characterization by methylome-analysis. While the histomorphological best-fit was CNS-neuroblastoma, unsupervised clustering was closest to ETMR. The CNAs did not match either diagnosis. Interestingly, the MGMT promoter was methylated, which has only been rarely reported in embryonal tumors, and despite indicating sensitivity to temozolomide and lomustine, has not been predictive of survival ${ }^{8,9}$. Overall, this suggested unique biology, and that this novel entity is not currently represented in the classifier database.

As the use of methylome-based tumor classification is currently limited to specialized centers in Europe, North 
America and Australia, it is not surprising that rare tumor subsets from other demographic populations may be under-represented. Limitations have been apparent for rare tumors like NTRK-fused gliomas ${ }^{10}$ and those arising from germline defects like mismatch-repair deficiency ${ }^{11}$. Twinning initiatives and collaboration between centers of excellence in the West and the large-volume centers in developing countries are needed to advance this diagnostic tool and benefit the entire spectrum of children with CNS tumors.

Source of funding: AD is supported by the St. Baldrick's Foundation International Scholar Award (with generous support from the Team Campbell Foundation; Grant number: 697257) and the SickKids Research Training Center Clinician-Scientist Training Program (Fall 2019 award).

Acknowledgements: We are grateful to Dr Martin Sill (DKFZ, Heidelberg) for analysing and confirming that methylation profile similar to the index tumor was not identified in their database.

Consent: Informed consent was obtained from the parents for publishing this case report.

\section{REFERENCES}

1. Sturm D, Orr BA, Toprak UH, et al. New Brain Tumor Entities Emerge from Molecular Classification of CNS-PNETs. Cell. 2016;164:1060-1072.

2. Louis DN, Perry A, Wesseling P, et al. The 2021 WHO Classification of Tumors of the Central Nervous System: a summary. Neuro-Oncol. 2021;23:1231-1251.

3. Holsten T, Lubieniecki F, Spohn M, et al. Detailed Clinical and Histopathological Description of 8 Cases of Molecularly Defined CNS Neuroblastomas. J Neuropathol Exp Neurol. 2021;80:52-59.

4. Łastowska M, Trubicka J, Sobocińska A, et al. Molecular identification of CNS NB-FOXR2, CNS EFTCIC, CNS HGNET-MN1 and CNS HGNET-BCOR pediatric brain tumors using tumor-specific signature genes. Acta Neuropathol Commun. 2020;8:105.

5. Korshunov A, Okonechnikov K, Schmitt-Hoffner F, et al. Molecular analysis of pediatric CNS-PNET revealed nosologic heterogeneity and potent diagnostic markers for CNS neuroblastoma with FOXR2activation. Acta Neuropathol Commun. 2021;9:20.

6. Lambo S, Gröbner SN, Rausch T, et al. The molecular landscape of ETMR at diagnosis and relapse. Nature. 2019;576:274-280.

7. Raghuram N, Khan S, Mumal I, Bouffet E, Huang A. Embryonal tumors with multi-layered rosettes: a disease of dysregulated miRNAs. J Neurooncol. 2020;150:63-73.

8. Faoro D, von Bueren AO, Shalaby T, et al. Expression of $\mathrm{O}^{6}$-methylguanine-DNA methyltransferase in childhood medulloblastoma. J Neurooncol. 2011;103:59-69.

9. Oh J, Bilbao JM, Tsao MN, et al. Recurrent PNET with MGMT methylation responds to temozolomide. Can J Neurol Sci J Can Sci Neurol. 2009;36:654-657.

10. Torre M, Vasudevaraja V, Serrano J, et al. Molecular and clinicopathologic features of gliomas harboring NTRK fusions. Acta Neuropathol Commun. 2020;8:107.

11. Dodgshun AJ, Fukuoka K, Edwards M, et al. Germline-driven replication repair-deficient high-grade gliomas exhibit unique hypomethylation patterns. Acta Neuropathol. 2020;140:765-776.

\section{FIGURE LEGEND}

Fig.1. (A) MRI shows enhancing diffusion restricted fairly large well defined T2/Flair heterogeneously hyperintense mass in left parietal lobe with effacement of adjacent sulci and gyri and mild midline shift. (B) Histology demonstrated neuropils surrounded by small blue round cell tumors in diffuse sheets and rosettes surrounded by single layer of small round blue cells. (C)Synaptophysin staining of sheets of small round blue cells. Other IHC stains demonstrated (not shown) GFAP patchy +, NeuN patchy +, Olig2 -, Chromogranin -, LIN28 -, INI-1 retained, Desmin -, EMA -. Ki67 was $\sim 20 \%$. (D) Unsupervised clustering 
(tSNE plot; Supplement: Methods) showed that the tumor grouped closest with ETMR (red arrow). (E) Copy number aberrations plot derived from methylation profiling. (F) Focused analysis (using the conumee package; Supplement: Methods) revealed no structural variation at C19MC region. (G) The MGMT promoter was methylated.

[Abbreviations in figure : FLAIR: Fluid-attenuated inversion recovery. DWI: Diffusion weighted imaging. MB: Medulloblastoma. EPN: Ependymoma. PF: Posterior fossa. HGG: High-grade glioma. GBM: Glioblastoma. MES: Mesenchymal. MID: Midline. K27M: Diffuse midline glioma, K27-altered. G34R: Diffuse hemispheric glioma, G34-mutant. PXA: Pleomorphic xanthoastrocytoma. Ana-PA: Anaplastic pilocytic astrocytoma. ATRT: Atypical teratoid rhabdoid tumor. ETMR: Embryonal tumor with multilayered rosettes. HGNET: High-grade neuroepithelial tumor. NB: Neuroblastoma. EWS: Ewing sarcoma. EFT: Ewing family tumor. MGMT: $\mathrm{O}(6)-$ Methylguanine-DNA-methyltransferase.]

\section{SUPPLEMENT: METHODS:}

The pathology was reported at Tata Medical Center, Kolkata (LZ and PR). Review and confirmation of the pathology $(\mathrm{CH})$, and further molecular testing were performed at the Hospital for Sick Children (SickKids), Toronto. DNA methylation profiling was done using the Illumina Human Methylation $850(850 \mathrm{k})$ array. The idat files were uploaded on the molecular neuropathology 2.0 website (https://www.molecularneuropathology.org/mnp) and were analysed on the brain tumor classifier versions $11 \mathrm{~b} 4 \mathrm{v} .3$ and 12.3v.1. Further analysis, including the unsupervised clustering, was performed in R v.4.0.3. Unprocessed .idat files for control methylation tumor classes were downloaded from the NCBI Gene Expression Omnibus (GEO), under accession number GSE73801, as published before ${ }^{1}$. The ChAMP, minfi, limma and Rtsne packages were utilized for downstream analyses. Raw data were obtained from idat files and normalized using the single sample normal-exponential out-of-band method (ssNoob). Probes on the X and $\mathrm{Y}$ chromosomes were removed, along with probes with a bead count of less than 3 , those with a detection $\mathrm{p}$ value above 0.01, and probes at known SNP locations or with multiple binding locations as previously reported by our group. ${ }^{11}$ T-distributed stochastic neighbor embedding (t-SNE) analysis using the Rtsne package applied the following nondefault parameters: theta $=0$, pca $=\mathrm{F}$, max iter $=7500$, and perplexity $=15$. Copy number alterations of genomic segments were inferred from the methylation array data based on the R-package conumee after additional baseline correction (https://github.com/dstichel/conumee). Visualization was performed using the ggplot2 and the plots were edited for aesthetics using Adobe Illustrator v.25.4.1. 


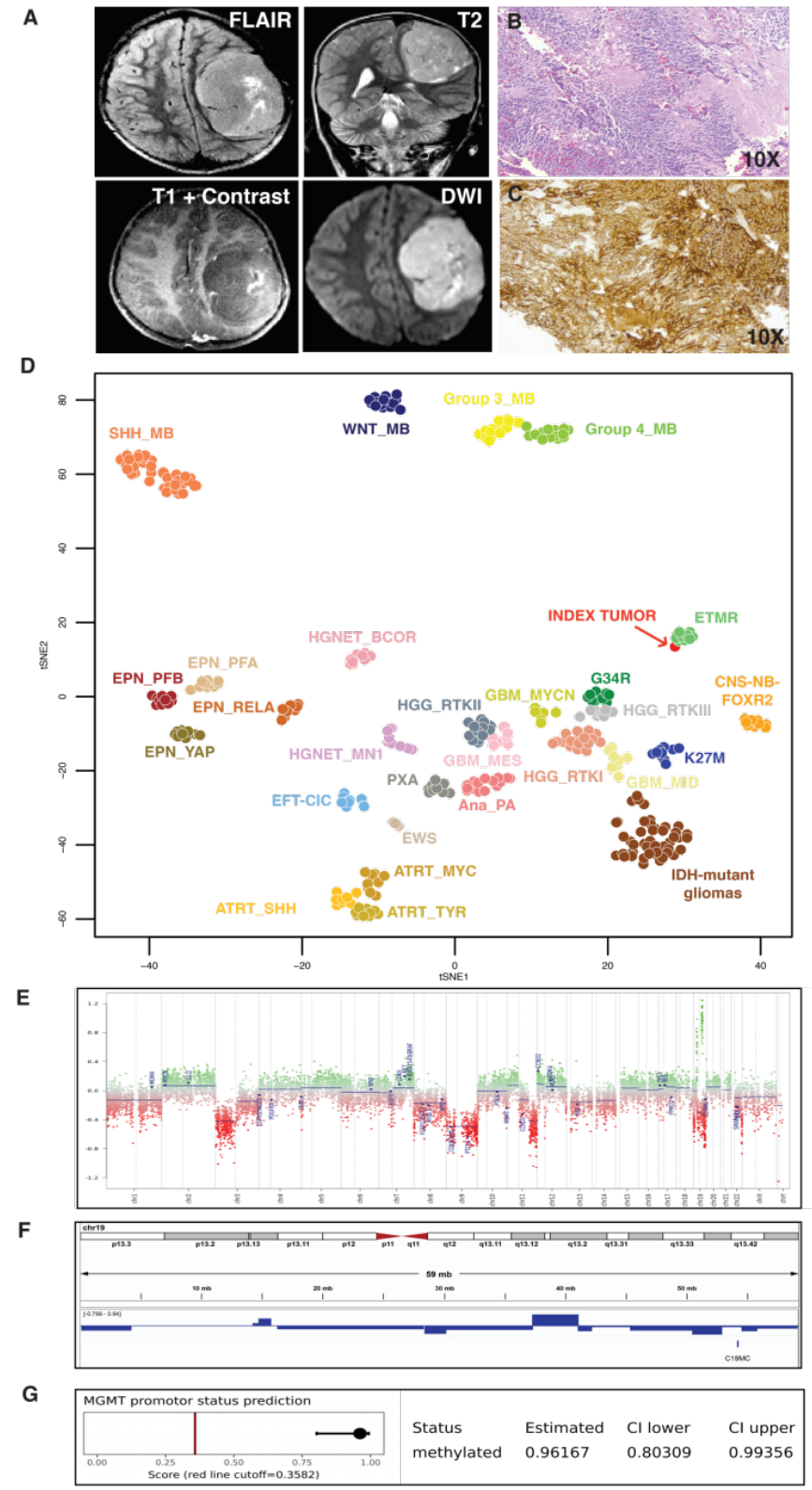

